\title{
OPTICAL CORE-SHELL MODEL FOR NANO-DELIVERY APPLICATIONS
}

\author{
Ana J. Šetrajčić-Tomić1, Dušan Lj. Džambas², Ljubiša D. Džambas3, Jovan P. Šetrajčićc* \\ ${ }^{1}$ University of Novi Sad, Medical Faculty, Department of Pharmacy, Serbia \\ ${ }^{2}$ University of Novi Sad, Medical Faculty, $5^{\text {th }}$ year Regular Student, Serbia \\ 3 University of Novi Sad, Medical Faculty, Department of Dentistry, Serbia \\ ${ }^{4}$ University of Novi Sad, Faculty of Sciences, Department of Physics, Serbia
}

\begin{abstract}
In this paper, we will analyze the application of nanomaterials in biomedicine, that is to say, we will present the recent accomplishments in basic and clinical nanomedicine. Achieving the full potential of nanomedicine may be years or even decades away; however, potential advances in drug delivery, diagnosis, and the development of nanotechnology-related drugs are starting to change the landscape of medicine. Site-specific targeted drug delivery (made possible by the availability of unique delivery platforms, such as dendrimers, nanoparticles and nanoliposomes) and personalized medicine (result of the advance in pharmacogenetics) are just a few concepts on the horizon of research. In this paper, we have especially analyzed the changes in basic physical properties of sphericalshaped nanoparticles that can be made in several (nano)layers and have, at the same time, multiple applications in medicine. The subject of the research in this paper includes the modeling of nanomaterials in the field of pharmaceutical technology for biomedical application. This includes a very precise encapsulated drug delivery on the exactly defined place in the human tissue or organ and the disintegration of the capsule - drug carrier, so that the medicament can start producing its effect. The goal of multidisciplinary researches with biocompatible molecular nanomaterials is to find the parameters and the possibilities to construct boundary surfaces that will, in interaction with the biological environment, create such properties of nanolayers that can be conveniently used for layers of drug carrier capsules, biochips and biomarkers. These layers should demonstrate a controlled disintegration of structure, better dielectric properties, discrete luminescence and appropriate bioporosity, as all of these are the requirements of contemporary nanomedicine.
\end{abstract}

Key words: Ultrathin films, optical biomaterials, nanomedicine, core-shell model, drug deliveries

DOI: $10.21175 / \operatorname{RadProc} .2016 .39$

\section{INTRODUCTION}

The development of biomedical science is directly related to the development of natural and mathematical sciences, especially materials science. Enormous resources are invested in the research that will lead to the solutions for many deadly diseases and, in the end, it is impossible not to put a completely logical question: whether the results of modern research justify the invested resources $[1,2]$ ?

Let us consider in what ways we can make a contribution to research in science. One approach is experimental, while the other is theoretical. There are big differences in the popularity of these approaches, yet both are said to have advantages and disadvantages, as well.

There are two aspects of the theoretical approach. One uses computer resources, while the other uses analytic (literally speaking, paper and pencil) ones. The aim of our research group is to understand, within materials science, the essential mechanisms of transport processes in low-dimensional systems, so we chose an analytical approach with the ambition that in the very near future, we can study with the help of computer resources too. Our method of choice is the Green's function, which we use in the field of molecular crystals.

In this paper, we will analyze the application of nanomaterials in biomedicine, that is to say, we will present the recent accomplishments in basic and clinical nanomedicine. Numerous novel nanomedicinerelated applications are under development or are in a research phase, and the process of converting basic research in nanomedicine into commercially viable products will be long and difficult. Achieving the full potential of nanomedicine may be years or even decades away; however, potential advances in drug delivery, diagnosis, and the development of nanotechnology-related drugs are starting to change the landscape of medicine. Site-specific targeted drug delivery (made possible by the availability of unique delivery platforms, such as dendrimers, nanoparticles and nanoliposomes) [3-5] and personalized medicine (the result of the advance in pharmacogenetics) [6-8] are just a few concepts on the horizon of research.

\footnotetext{
*bora@df.uns.ac.rs
} 


\section{DIELECTRICAL PROPERTIES OF MOLECULAR CRYSTALS}

The basic idea was to examine the exciton subsystem in the bulk systems, then in quasi-twodimensional ones, such as ultra-thin films of molecular crystals, and then to compare the results to get to the micro-theory which would decipher the mechanisms of the optical properties of the observed system [9,10].

Since we are interested in the optical properties of the molecular crystals, it is essential to observe the exciton subsystem. If we use the model of Frenkel's excitons with low concentrations, then the exciton subsystem in the harmonic approximation can be described by the following Hamiltonian [9]:

$$
H=\sum_{\vec{n}} \Delta_{\vec{n}} B_{\vec{n}}^{+} B_{\vec{n}}+\sum_{\vec{n}, \vec{m}} X_{\vec{n} \vec{m}} B_{\vec{n}}^{+} B_{\vec{m}},
$$

where $B_{\vec{n}}^{+}$and $B_{\vec{n}}$ are creation and annihilation operators of an exciton at the node $\vec{n}$ of a crystal grid, $\Delta_{\vec{n}}$ is the energy of an isolated exciton in that node, and $X_{\vec{n} \vec{m}}$ are matrix elements of excitons' transfer from node $\vec{n}$ to node $\vec{m}$. In the model, it is assumed that the exciton energy at the node is $\sim 10^{2}$ times bigger than the energy of its transfer.

Theoretical - quantum-mechanical analysis of the modeling system can be accomplished by solving the Schrödinger's equation, or by Heisenberg's equations of motion with the use of DFT approach. Based on these methods, studies considering simulations emerged - using an appropriate software package [1113]. This package uses Schrödinger's (state-of-art) approach, employs wave functions to set the BetheSalpeter's equation and solves it using the numericalgraphical method. We have developed our own computer application which can test boundary manipulation in order to obtain a reasonable fundamental understanding of the material properties and compare the results with the experimental measurements. At the beginning, we applied (i.e. tested) this computerized method on simple examples. On the other hand, with such simplification, we could recognize and individually extract the real and relevant factors that affect the changes of physical properties, thus determining their real intensity.

We opted for the tested and the adapted methods of the Green's function [9], using the spectrum and the states of elementary excitations which could be easily determined, as well as the equilibrium characteristics of the system [14]. The method of two-time temperature Green's functions has been chosen for the microtheoretical analysis because of its benefits - the real parts of poles of Green's functions define the energies of elementary excitations (from which the law of dispersion is obtained). If the Hamiltonian has a harmonic form, the Green's function has real poles only. Green's function $G_{\vec{n} \vec{m}}(t)=\left\langle\left\langle B_{\vec{n}}(t) \mid B_{\vec{m}}^{+}(0)\right\rangle\right\rangle$ satisfies the certain equation of motion and by using the complete time and space Fourier's transformation, we may obtain a form of Green's function:

$$
G_{\vec{k}}(\omega)=\frac{1}{2 \pi i} \frac{1}{\omega_{\vec{k}}-\omega}
$$

and from its pole we can also obtain the desired law of dispersion of excitons,

$$
\hbar \omega_{\vec{k}}=\Delta+2\left(X_{x} \cos a_{x} k_{x}+X_{y} \cos a_{y} k_{y}+X_{z} \cos a_{z} k_{z}\right)
$$

which may be written in the following non-dimensional form:

$$
E_{\vec{k}} \equiv \frac{\hbar \omega-\Delta}{|X|}=F_{x y}+R_{z},
$$

where:

$$
F_{x y} \equiv 2\left(\cos a k_{x}+\cos a k_{y}\right), R_{z} \equiv 2 \cos a k_{z} .
$$

Eventually, through a general expression [9] for relative dynamic dielectric permittivity,

$$
\varepsilon^{-1}(\omega)=1-2 \pi i S[G(\omega)+G(-\omega)]
$$

we can obtain the expression for dynamic permittivity of bulk:

$$
\varepsilon \equiv \varepsilon(\omega)=\left(1+\frac{2 S \omega_{\vec{k}}}{\omega^{2}-\omega_{\vec{k}}^{2}}\right)^{-1}
$$

where $S$ is a parameter of the inner structure. Figure 2 shows dynamical permittivity of the bulk-structure.

\section{ULTRATHIN MOLECULAR LAYER}

Our model of a crystal nanofilm assumes an ultrathin film confined by two parallel planes that are infinite in the $x$ and $y$ directions, while in the $z$ direction the film has a final thickness $L=N a$. We will observe a symmetrical nano-film (Figure 1), which may be made by controlled reaching of massive specimens. Due to the presence of boundary areas, the energies of excitons in the nodes and the energy transfers between boundary areas and neighboring areas are perturbed, where parameter $d$ defines perturbation at the node of boundary areas, and the parameter $x$ is a perturbation of transfer in boundary layers along the $z$-direction.

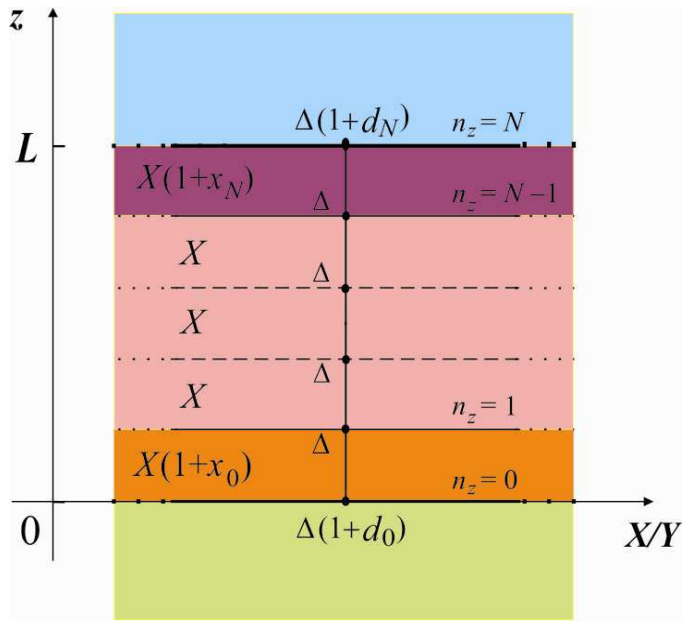

Figure 1. Ultrathin film model 
Now, for ultrathin films, we will use the same procedure for their determination. In the case of a nanofilm, we perform full time and partial space Fourier transformation of these equations, because we have limited structure along $z$-direction. Figure 2 shows dispersion laws for non-perturbed (ideal) symmetric five-layered film (reduced energies are used again). Full lines show energy levels of excitons in the observed film, while broken lines show boundaries of continual energy zone of excitons in a bulk. At the first sight it is possible to see the absence of zero values and discretion of exciton energies in a film. The number of possible states corresponds to the number of crystallographic levels in this film along the $z$-axis.

For higher values of the parameter $d$, energy levels are shifted to higher energies and at one point leaving the boundaries of the bulk. This energy is called localized or TAM states. Figure 2 gives an example of a very perturbed $(d=0,3, x=2,0)$ symmetric fivelayered film.

The most probable localized states are at the very boundaries of a film or in the boundary layers of that film. Inside the film only bulk states are present and their probability, in comparison to localized states, is much smaller.
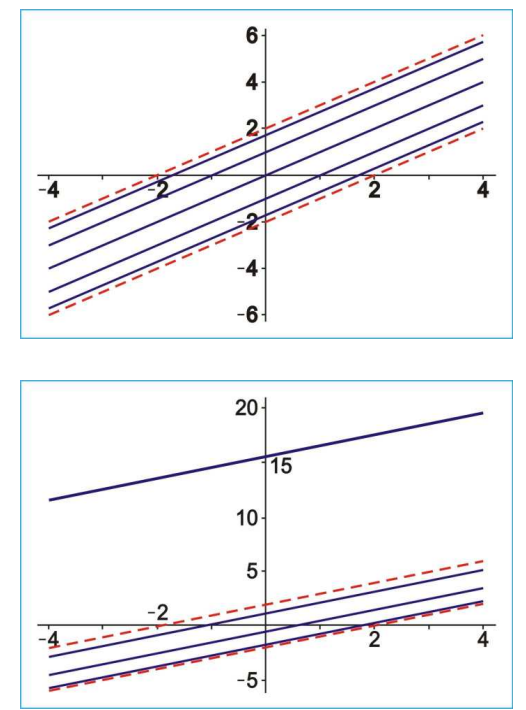

Figure 2. Dispersion law of ultrathin films

After the application of Green's functions, one can obtain the expression which represents the relative dynamical permittivity as a function of the frequency of initial electromagnetic excitation. Again it is possible to change the values of perturbation parameters.

Our scope of research is the optic properties of the observed model example. The refraction $(n)$ and absorption ( $\kappa$ ) indices are usually defined in the literature [10] by permittivity term: $\sqrt{\varepsilon}=n+i \kappa$. Introducing the complex frequency: $\omega=\omega+i \nu$ in expression for permittivity (7), we get complex permittivity: $\varepsilon=\mathcal{E}^{\prime}+i \varepsilon^{\prime}$. Based on this, we can find the expression for absorption and refraction indices in the following form:

$$
\begin{aligned}
& \kappa_{n_{z}}(\omega)=\sqrt{\frac{\varepsilon_{n_{z}}^{\prime}(\omega)}{2}\left\{\sqrt{1+\left[\frac{\varepsilon_{n_{z}}^{\prime \prime}(\omega)}{\varepsilon_{n_{z}}^{\prime}(\omega)}\right]^{2}}-1\right\}} ; \\
& n_{n_{z}}(\omega)=\sqrt{\frac{\varepsilon_{n_{z}}^{\prime}(\omega)}{2}\left\{\sqrt{1+\left[\frac{\varepsilon_{n_{z}}^{\prime \prime}(\omega)}{\varepsilon_{n_{z}}^{\prime}(\omega)}\right]^{2}}+1\right\}} .
\end{aligned}
$$

The reflection $(r)$ and transparency $(t)$ indices are defined [10] by the refraction and absorption ones:

$$
\begin{aligned}
& r_{n_{z}}(\omega)=\frac{\left[n_{n_{z}}(\omega)-1\right]^{2}+\kappa_{n_{z}}^{2}(\omega)}{\left[n_{n_{z}}(\omega)+1\right]^{2}+\kappa_{n_{z}}^{2}(\omega)} ; \\
& t_{n_{z}}(\omega)=1-n_{n_{z}}(\omega)-\kappa_{n_{z}}(\omega) .
\end{aligned}
$$

From this and the expressions (8) and (7), one can see that dielectric and all optical characteristics of the film depend on the position of the film-layer.

By numerical calculations, we have analyzed dynamical permittivity and optical indices behavior on plane position and on values of boundary parameters, and calculated that. The number of resonant peaks directly depends on the film width, i.e., on the number of layers $N$ (there $N=3$ ). The general rule is that the number of resonance peaks decreases inside the layers of the film, although with the influence of perturbation parameters $d_{0, N} \in[-0.25,+0.25]$ and $x_{0, N} \in[-0.9,+$ 2.0] that rule can be broken and in that case the (maximal) number of peaks is $N+1 \equiv 4$, with the possibility of some resonance peaks disappearing, i.e. overlapping or vanishing.

Subsequently, we continued with the research of the same structures and investigated the influence of boundary parameters on optical properties of these structures, for different values of perturbation parameters. Graphics on Figure 3 show some results for representative cases for three-layered asymmetrically perturbed film, with: $d_{0}=-0,15, d_{N}=$ 0,$15 ; x_{0}=-0,75$ and $x_{N}=0,75$.

Relative dynamic permittivity of the bulk samples of molecular crystals have a complete absorption zone in the infrared (IR) area [9], corresponding to the absorption of all IR rays with the relative frequency $f \in$ $(43.5,47.5)$. This means that the optical indices of the bulk sample, in this frequency interval, have the following values: $\kappa_{\mathrm{b}}=1, r_{\mathrm{b}}=\tau_{\mathrm{b}}=0$ and $n_{\mathrm{b}}=n$. On the contrary, in ultrathin films, only discrete absorption lines will occur, and their number corresponds to the number of possible exciton states. The distribution and intensity of absorption lines depend on the value of the boundary parameters!

From the presented graphics, one can see that the film-structures have discrete and selective optical properties, that there is a maximum of 4 discrete absorption lines, equal to the number of possible (macro) quantum states of excitons through filmthickness [10,15].

In the case of bulk, there is a completely clear absorption zone, while in the case of films, there are $\leq 4$ absorption peaks corresponding to the resonant lines.

Refraction indices have jumps on the places where the absorption index changes: for the bulk, that is happening on the borders of the absorption zone, while 
the film has $1-4$ corresponding, not very narrow peaks.

Reflection for the bulk is only out of the borders of the absorption zone; for films, reflection is happening partially at each crystallographic plane of the film.

The most interesting result is definitely nontransparency of the molecular bulk, but when it is thinned to the film of nano-size, it becomes transparent, except for the maxima 4 discrete peaks which correspond to a combination of absorption and reflection peaks of the film.

From these expressions, one can see that dynamical absorption and refraction indices, as well as relative permittivity, depend on the position of the film-layer $\left(n_{z}\right)$ and on the perturbation parameters at and within the shall boundary layers. Graphics in Figure 3 show some results for representative cases, which are analogous to the ones in permittivity.

Since the emission and absorption spectra can be experimentally recorded, but only for the whole film, we have determined these optical characteristics (indices of absorption and refraction) not only for the particular layers but for the whole shall as well, and in the normal direction on the boundary surface of the shall (or radial from core center), where the changes of these values occur in relation to their bulk values.

Because the index of absorption is a part of the energy/intensity electromagnetic radiation which is absorbed by the film in relation to the total incident energy/intensity of the radiation that got to the film, it can be concluded that the overall indices of absorption $\left(\kappa_{F}\right)$, reflection $\left(r_{F}\right)$ and transparency $\left(t_{F}\right)$ for the whole film could be acquired by simply adding up adequate indexes on individual planes, i.e.

$$
\phi_{F}(\omega)=\sum_{n_{z}} \phi_{n_{z}}(\omega) ; \quad \phi \equiv(\kappa, r, \tau) .
$$

Of course, this summation means that the addition is done strictly by the individual, i.e., specific frequencies, because it is a question of values of relative dynamic quantities.

In contrast, refractive indexes present the ratios of appropriate speeds, and therefore additivity and simple sum cannot be applied to them. It is not difficult to show that [16-20], during the formation of the refractive index of the whole film, the rule of the sum of reciprocal values must be applied in the following form:

$$
\frac{N}{n_{F}(\omega)}=\sum_{n_{z}} \frac{1}{n_{n_{z}}(\omega)} .
$$

Based on the last two formulas, we numerically determined the dependence of the absorption index and the refraction index for the whole film. The results of these calculations are presented in Figure 3.

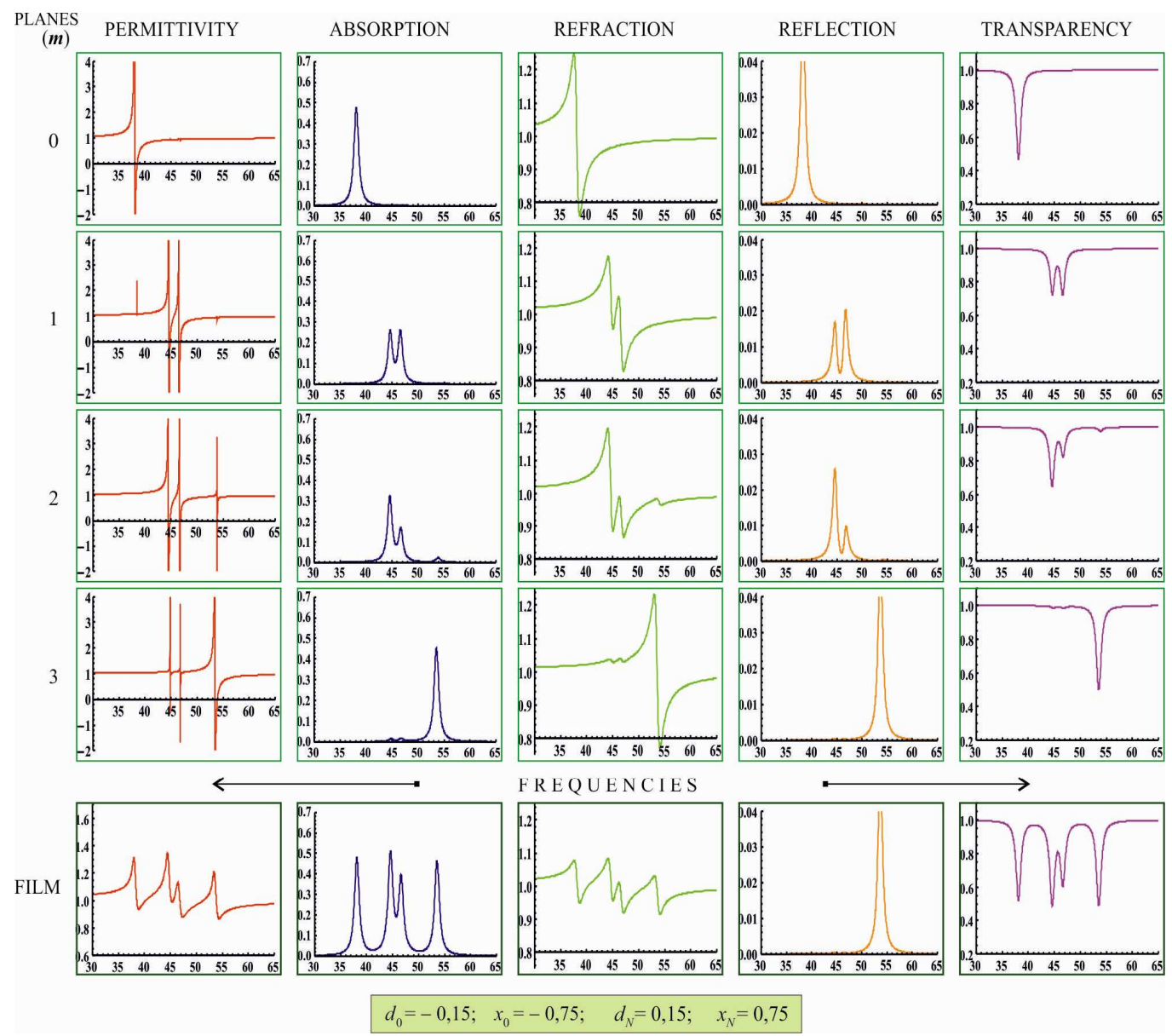

Figure 3. Relative permittivity and relevant optical indices of a highly perturbed non-symmetrical five-layered film 


\section{Potential RESEARCH OF CORE SHELL NANOMODELS}

Our research group believes that the methodology used to analyze the optical properties of nano-films can be used for theoretical studies of materials with transfer character to be used in nano-pharmacy. We are primarily thinking of core shell models under which the systems include a spherical shape. The basic idea of a core shell model in biomedicine is to fill the interior of hollow nano-spheres with the active component of pharmaceuticals. The role of hollow spheres measuring up to tens of nanometers is transport. Thanks to the dimensions and properties of hollow nano-spheres, they are ideal candidates to be hosts for the active components of pharmaceuticals, which cannot be created by conventional methods without serious consequences transferred to the target place. Take the example of ketoprofen, a good conventional nonsteroidal anti-inflammatory agent, the oral intake of which can lead to ulcer. The second but unconventional way of entering the body is through polymeric nano-particles such as PDLLA. The result is the introduction of pharmaceuticals as a cream, which would be rubbing in determined places what would transfer it, rather than orally.

The main advantage of the theoretical approach is the essential knowledge of the mechanisms that allow us to comprehend the experimental conditions that must be fulfilled to get the desired results. The results achieved up to now by our research group in application of the Green's function method on flat ultrathin films are promising for applications in the frame of core-shell models. This paper presents the review of our current achievement in the field of theoretical physics of ultrathin films and possible ways to materialize the same in the field of nanopharmacy.

It is certain that the solution to the problems of delivery and transport of pharmaceuticals to different parts of the body lies in the achievements of materials science. In this sense, nano-materials and hollow nanospheres are the materials which will drive attention in the future even more than now. For positive results in this area, experimental methods are used much more. Even those theoretical mainly represent the results obtained using computing resources, as opposed to analytical methods [9,13].

Our goal is the use and adaptation of analytical methodology applied to flat ultrathin films on the spherical systems that look as if composed of a certain number of ultrathin films [21].

What could the research of the material look like if it could be modeled as a core shell [22]? If we performed the research of the material, in the most general case we could examine the three major subsystems: phonon, electronic and exciton. In general, the results related to the phonon subsystem would show us what the mechanical properties of the materials would be, the electronic sub-system would give us conductive properties, while the exciton subsystem would be "responsible" for the optical properties.

As we already operate with the testing methodology of the specified subsystem, the next step would be adjusting the methodology to the spherical systems, as the hollow nanospheres are, that might be carriers of pharmaceuticals. Namely, a nanosphere can be seen just as a system composed of a number of ultra-thin films, Figs. 4 and 5. So, in this way, the results connected to ultrathin films could be used. Of course, the transition from flat to spherical ultrathin films is not a trivial problem. The first step is to create an adequate Fourier transformation, but the real problem is how to include a flat deformation of a thin film that would emerge. Further research of some subsystem would follow, primarily phonon, since the mechanical properties in terms of application are the most important. The research of conductive and optical properties may lead to the knowledge of how the holders of pharmaceutical nanoparticles can be induced, i.e., how they can be manipulated (e.g. target more).

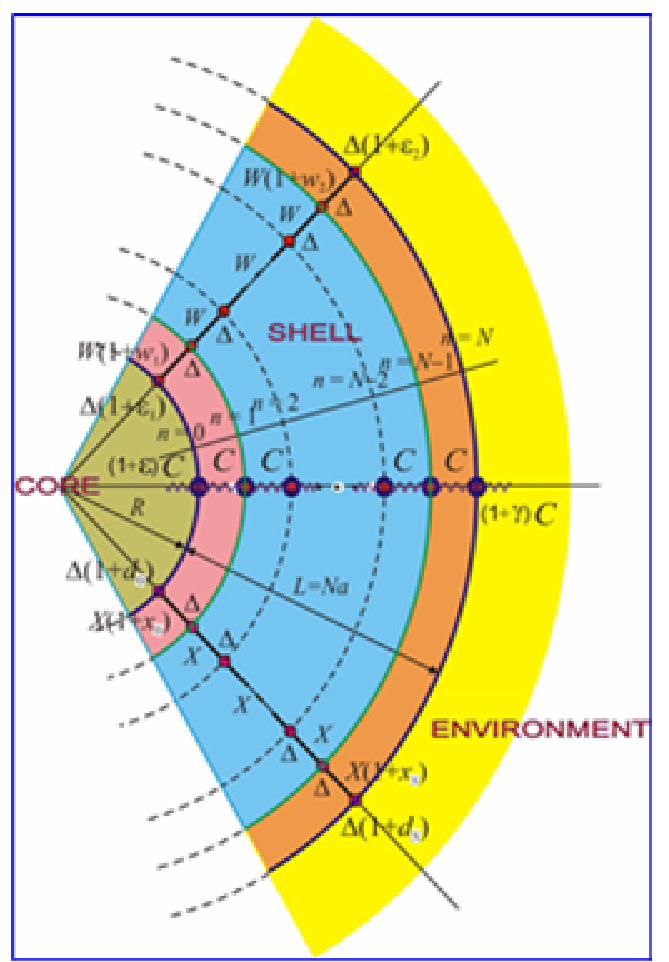

Figure 4. Model of spherical layered ultrathin films

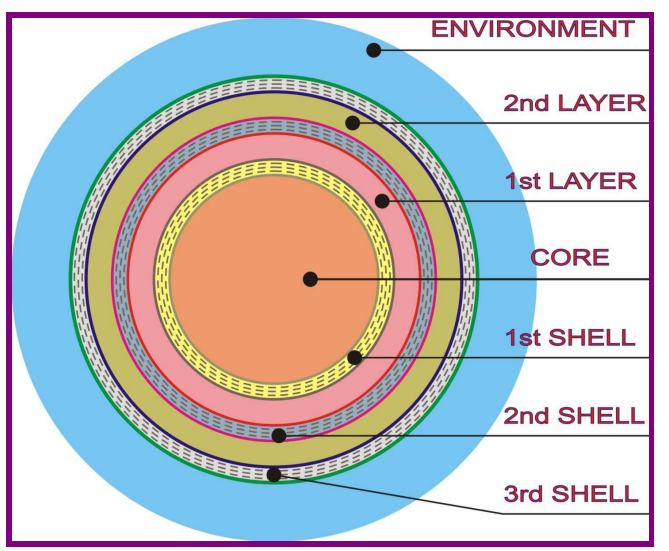

Figure 5. Spherical multi-layered core-shell model

As it is very hard to reach the analytical solutions that are related to the dynamics of subsystems, for a start, a potential approach might be to use computer resources, i.e., to create a simulation that would lead to some general results and could indicate the solution. 


\section{CONCLUSION}

Our model of a crystal nanofilm assumes an ultrathin film confined by two parallel planes that are infinite in the $x$ and $y$ directions, while in the $z^{-}$ direction the film, it has a final thickness $L=N a$. We will observe a symmetrical nano-film (Figure 1), which may be made by controlled reaching of massive specimens. Due to the presence of boundary areas, the energies of excitons in the nodes and the energy transfers between boundary areas and neighboring areas are perturbed, where the parameter $d$ defines perturbation at the node of boundary areas, and the parameter $x$ is the perturbation of transfer in boundary layers along the $z$-direction.

It is certain that the solution to the problems of delivery and transport of pharmaceuticals to different parts of the body lies in the achievements of materials science. In this sense, nano-materials and hollow nanospheres are the materials which will get attention in the future even more than now. For positive results in this area, experimental methods are used much more. Even those theoretical mainly represent the results obtained using computing resources, as opposed to analytical methods.

Our goal is the use and adaptation of analytical methodology applied to flat ultrathin films on the spherical systems that look as if composed of a certain number of ultrathin films.

Acknowledgements: The paper is a part of the research done within the projects $O N-171039$ and $T R$ 34019 (Ministry of Education, Sciences and Technological Development of the Republic of Serbia), and project 19/6-020/961-16/15 (Ministry of Science and Technology of the Republic of Srpska) as well as the project 114--451--972 (Provincial Secretariat for Science and Technological Development of Vojvodina).

\section{REFERENCES}

1. K.J. Morrow, R.Bawa, C. Wei, "Recent Advances in Basic and Clinical Nanomedicine," Med .Clin. N. Am., vol. 91, no. 5 , pp. 805-843, 2007

2. Drug Delivery Nanoparticles - Formulation and Characterization, vol. 191, Y. Pathak, D. Thassu, Eds., New York (NY), USA: Informa Healthcare, 2009

3. H.E. Schaefer, Nanoscience - The Science of the Small in Physics, Engineering, Chemistry, Biology and Medicine, Berlin, Germany: Springer, 2010

4. M.A. Greenwood, "Are Quantum Dots on the Brink of Their Big Break?” Phot. Spectra, May 2007

5. E.A. Murphy et al., "Nanoparticle-Mediated Drug Delivery to Tumor Vasculature Suppresses Metastasis," PNAS, vol. 105, no.27, pp. 9343-9348, 2008

6. W.C.W. Chan, "Bionanotechnology Progress and Advances," Biol. Blood Marr. Transpl., vol. 12, no. 1, suppl. 1, pp. 87-91, Jan 2006

7. Y. Pathak, "Recent Developments in Nanoparticulate Drug Delivery Systems," in Drug Delivery Nanoparticles - Formulation and Characterization, vol. 191, Y. Pathak, D. Thassu, Eds., New York (NY), USA: Informa Healthcare, 2009, sec. 1, pp. 1-15
8. H. Devapally, A. Chakilam, M.M. Amiji, "Role of Nanotechnology in Pharmaceutical Development," $J$. Pharm. Sci., vol. 96, no. 10, pp. 2547-2565, Oct. 2007

9. J.P. Šetrajčić, "Exact Microtheoretical Approach to Calculation of Optical Properties of Ultralow Dimensional Crystals," arXiv:cond-mat/eprint: 1004.2387 (Cornell Univ. Lib.), Apr. 2010

10. J.P. Šetrajčić et al., "Absorption Features of Symmetric Molecular Nanofilms," in Proceedings 27th MIEL, Niš, Serbia, 2010, pp. 127-130

11. S.Y. Quek, J.B. Neaton, M.S. Hybertsen, E. Kaxiras and S.G. Louie, "First-principles Studies of the Electronic Structure of Cyclopentene on Si(O01): Density Functional Theory and GW Calculations," Phys. Stat. Solidi (b), vol. 243, no. 9, 2048-2053, July 2006

12. G. Samsonidze, M. Jain, J. Deslippe, M.L. Cohen and S.G. Louie, "Simple approximate Physical Orbitals for GW Quasiparticle Calculations," Phys. Rev. Lett., vol. 107, p. 186404, Oct. 2011

13. J. Deslippe, G. Samsonidze, D.A. Strubbe, M. Jain, M.L. Cohen and S.G. Louie, "BerkeleyGW: A Massively Parallel Computer Package for the Calculation of the Quasiparticle and Optical Properties of Materials and Nanostructures," Comput. Phys. Commun., vol. 183, no. 6 pp. 1269-1289, June 2012

14. G. Mahan, Many-Particle Physics, 2nd ed., New York (NY), USA: Plenum Press, 1990

15. J.P. Šetrajčić et al., "Phonon Contribution in Thermodynamics of Nano-Crystalline Films and Wires," Acta Phys .Pol. A, vol. 115, no. 4, pp. 778-782, 2009

16. S.M. Stojković, D.Lj. Mirjanić, J.P. Šetrajčić, D.D. Šijačić, I.K. Junger, "Spectra and States of Electrons in Surface Perturbed Quantum Wires,” Surf. Sci., vol. 477, no. 2-3, pp. 235-242, Apr. 2001

17. D.I. Ilić, S.M. Vučenović, S.K. Jaćimovski, V.M. Zorić and J.P. Šetrajčić, "Phonon Spectra and Thermodynamics of Crystalline Nanowires," in LowDimensional Materials Synthesis, Assembly, Property Scaling, and Modeling, vol. 50, M. Shim, M. Kuno, X-M. Lin, R. Pachter and S. Kumar, Eds., San Francisco (CA), USA: Materials Res. Soc., 2007, pp. 1-6

18. I.D. Vragović, J.P. Šetrajčić and R. Scholz, "Quantum Size Effects in the Optical Properties of Organic Superlattices Containing 3,4,9,10 PTCDA," Eur. Phys. J. $B$, vol. 66 , no. 2, pp. 185-190, Nov. 2008

19. G. Samsonidze, F.J. Ribeiro, M.L. Cohen, and S.G. Louie, "Quasiparticle and Optical Properties of Polythiophene-Derived Polymers," Phys.Rev.B, vol. 90, no. 3, p. 035123, July 2014

20. Lj. Mirjanić, J.P. Šetrajčić, Lj. D.Džambas, V.D. Mirjanić, A.J. Šetrajčić-Tomić and V.M. Zorić, "Nanoscopic Biomaterials in Medicine, Dentistry and Pharmacy," in Procced. 1oth Int. Sci.-Practical Conf. "Research, Development and Application of High Technologies in Industry", Saint-Petersburg, Russia, 2010, pp. 97-107

21. J.P. Šetrajčić, D.Lj. Mirjanić, A.J. Šetrajčić-Tomić, S. Armaković and I.J. Šetrajčić, "Core-Shell Nanomodels for Biomedical Applications," in Proceed. Int. Conf. New Face of TMCR: Modern Techn., Quality and Innov., Vadul lui Voda, Moldova, 2011, pp. 545-548

22. J.P. Šetrajčić, A.J. Šetrajčić-Tomić, Lj.D. Džambas and I. Gušić, "Core-Shell Layered Models of Nanostructured Carriers for a Nano-Bio-Medical Applications," in Proceed. 3rd RAD, Budva, Montenegro, 2015, pp. 487494 